University of Nebraska - Lincoln

DigitalCommons@University of Nebraska - Lincoln

7-2007

\title{
Common Genome-Wide Patterns of Transcript Accumulation Underlying the Wing Polyphenism and Polymorphism in the Pea Aphid (Acyrthosiphon pisum)
}

\author{
Jennifer A. Brisson \\ University of Nebraska-Lincoln, jennifer.brisson@rochester.edu \\ Gergory K. Davis \\ Princeton University, gkdavis@princeton.edu \\ David L. Stern \\ Princeton University, stern@princeton.edu
}

Follow this and additional works at: https://digitalcommons.unl.edu/bioscifacpub

Part of the Life Sciences Commons

\footnotetext{
Brisson, Jennifer A.; Davis, Gergory K.; and Stern, David L., "Common Genome-Wide Patterns of Transcript Accumulation Underlying the Wing Polyphenism and Polymorphism in the Pea Aphid (Acyrthosiphon pisum)" (2007). Faculty Publications in the Biological Sciences. 42.

https://digitalcommons.unl.edu/bioscifacpub/42
}

This Article is brought to you for free and open access by the Papers in the Biological Sciences at DigitalCommons@University of Nebraska - Lincoln. It has been accepted for inclusion in Faculty Publications in the Biological Sciences by an authorized administrator of DigitalCommons@University of Nebraska - Lincoln. 


\title{
Common Genome-Wide Patterns of Transcript Accumulation Underlying the Wing Polyphenism and Polymorphism in the Pea Aphid (Acyrthosiphon pisum)
}

\author{
Jennifer A. Brisson (jbrisson2@unl.edu) $)^{1, *}$, Gregory K. Davis ${ }^{2, *}$ and David L. Stern² \\ ${ }^{1}$ University of Nebraska-Lincoln, Lincoln, NE, USA, ${ }^{2}$ Princeton University, Princeton, NJ, USA, "Joint first authors
}

\section{SUMMARY}

The pea aphid, Acyrthosiphon pisum, exhibits several environmentally cued polyphenisms, in which discrete, alternative phenotypes are produced. At low-density, parthenogenetic females produce unwinged female progeny, but at high-density females produce progeny that develop with wings. These alternative phenotypes represent a solution to the competing demands of dispersal and reproduction. Males also develop as either winged or unwinged, but these alternatives are determined by a genetic polymorphism. Winged and unwinged males are morphologically less distinct from each other than winged and unwinged females, possibly because males experience fewer trade-offs between dispersal and reproduction. To assess whether shared physiological differences mirror the shared morphological differences that characterize the wing polyphenism and polymorphism, we used a cDNA microarray repre-

\section{INTRODUCTION}

Many organisms can develop discrete alternative phenotypes, allowing them to adapt to environments that change in predictable ways. Examples include diet-induced trophic morphs of salamanders, seasonal morphs of butterflies, and seed-specific beak morphologies of finches (Collins and Holomuzki 1984; Smith 1993; Nijhout 2003). Examples such as these can be polyphenisms or polymorphisms. In a polyphenism, environmental cues lead to the use of alternative developmental pathways, whereas in a polymorphism, the chosen pathway is determined genetically. Although various theoretical treatments have suggested that polyphenism and polymorphism can evolve from each other (reviewed in West-Eberhard 2003), exactly how the two are related evolutionarily or mechanistically remains unclear. Species exhibiting both a polyphenism and a polymorphism for similar alternative phenotypes provide valuable models for studying senting an estimated $10 \%$ of the coding genome $(1,734$ genes) to examine differential transcript accumulation between winged and unwinged females and males. We identified several transcripts that differentially accumulate between winged and unwinged morphs in both sexes, the majority of which are involved in energy production. Unexpectedly, the extent of differential transcript accumulation between winged and unwinged morphs was greater for adult males than for adult females. Together, these results suggest not only that similar physiological differences underlie the polyphenism and polymorphism, but that male morphs, like females, are subject to trade-offs between reproduction and dispersal that are reflected in levels of transcript accumulation and possibly genome-wide patterns of gene regulation. These data also provide a baseline for future studies of the molecular and physiological basis of lifehistory trade-offs.

the relationship between the two phenomena because they avoid the confounding effects of genetic divergence and allow for examination of how they interact genetically.

In the pea aphid, Acyrthosiphon pisum, alternative wing morphs (i.e., winged and unwinged forms) are produced by a polyphenism in females and a polymorphism in males. The wing polyphenism occurs during the summer months when female pea aphids reproduce asexually with no genetic recombination, giving live birth to genetically identical daughters (Blackman 1987; Hales et al. 2002). Such females typically develop without wings, but produce winged daughters in response to crowding and possibly other cues such as poor host plant quality or interactions with other insects (Sutherland 1969; Muller et al. 2001). The mother perceives these cues and then transmits a wing-permissive signal to her embryos, which are competent to respond only within 48 hours before birth (Sutherland 1969). Winged daughters then disperse to less crowded host plants. 
Pea aphid males are produced asexually in the fall in response to shortened day length and lower temperatures (Via 1992). Males are XO and the male wing polymorphism is determined by a single unidentified locus on the $\mathrm{X}$ chromosome called aphicarus (api) (Smith and MacKay 1989; Caillaud et al. 2002; Braendle et al. 2005a). Winged and unwinged male siblings are thus genetically identical, except for their $\mathrm{X}$-chromosomes, which carry either the api-unwinged or the api-winged allele. It is unknown when during development api acts to determine morph type.

Although the environment cues the production of winged females, populations also harbor genetic variation for the propensity to respond to these cues (Markkula 1963; Hazell et al. 2005). One source of such variation is genetically linked to the api locus itself. Female clones carrying even one api unwinged allele produce large numbers of winged females compared with clones homozygous for the api-winged allele (Braendle et al. 2005b). Hence, the locus controlling the male wing polymorphism, api, is genetically linked to a locus or loci influencing female wing plasticity. If this linkage results from pleiotropy of a single gene, then the two phenomena may share aspects of their mechanisms of determination.

\section{Morphological and life-history differences between winged and unwinged aphids of both sexes}

Although we refer to the two morphs as simply winged and unwinged, they are in fact distinguished by systemic differences in morphology, behavior, and life history. In both sexes, winged morphs possess not only wings but also wing musculature, which is absent in unwinged morphs. Winged males and females also possess a more heavily sclerotized thorax and larger compound eyes relative to unwinged morphs (Takaoka 1973; Kring 1977; Kawada 1987; Miyazaki 1987). In both sexes, the behavior of wing morphs differs as well, with unwinged morphs being more sedentary (Sack and Stern 2007). Some morphological differences between wing morphs, however, are either absent or less pronounced in males relative to females. For example, although winged females possess ocelli and larger numbers of secondary rhinaria on their antennae when compared with unwinged females, both winged and unwinged males possess ocelli and the morphs differ less in the number of rhinaria (Kring 1977).

In insects, winged and unwinged morphs represent a tradeoff between dispersal and reproduction (Zera and Denno 1997). For example, in aphids, unwinged females reach maturity faster and have a higher fecundity than winged females (Dixon and Howard 1986). In some species, such as the pea aphid, winged females also histolyze their wing muscles at the start of reproduction following dispersal (Tsuji and Kawada 1987). Male life history is poorly studied, but it seems likely that trade-offs are less pronounced for male aphids because they devote proportionally fewer resources to gamete production than females and because winged males do not histolyze their wing muscles (Tsuji and Kawada 1987).
Differential transcript accumulation associated with the wing polyphenism and polymorphism

Because many morphological and behavioral differences between wing morphs are similar in females and males, we predicted that differences in physiology, reflected as differences in transcript accumulation, would also be similar. We also predicted that such differences would be less pronounced for males, as male morphs are less divergent morphologically and possibly experience fewer trade-offs. Here, we report genome-wide patterns of transcript accumulation in winged and unwinged females and males at two developmental stages. The results provide a window into the physiological differences and similarities that underlie the wing polyphenism and polymorphism. To our knowledge, this is the first study examining genome-wide patterns of differential transcript accumulation associated with wing dimorphism, a common phenomenon in insects. Readers should note the recent publication of a micro-array analysis of transcript accumulation in winged and unwinged adult females of the green peach aphid Myzus persicae (Ghanim et al. 2006).

\section{MATERIALS AND METHODS}

\section{Materials}

Aphids of the F1 clone, described previously in Braendle et al. (2005a), were used for all experiments in this study. Aphids were reared in an incubator at $19^{\circ} \mathrm{C}$ with 16 hours of light alternating with 8 hours of dark, in $15 \mathrm{~mm}$ Petri dishes, each with a leaf of Medicago arborea inserted into $3 \mathrm{ml}$ of 2\% agar containing $1 \mathrm{~g} / 1$ Miracle-Gro. To collect winged and unwinged females, female asexual aphids were reared at a low density (two to three aphids per plate) for three generations, initiating each generation with unwinged aphids, in order to eliminate grand-maternal effects. In the fourth generation, first instar females were reared in isolation to third-day adults and then placed in groups of 10 in a $10 \mathrm{~mm}$ Petri dish containing a moistened piece of Whatman paper. After 24 hours of crowding, the females were re-isolated in dishes with leaves. Progeny were removed on a daily basis and placed in individual dishes where they were monitored daily for molting. From these, winged females (identified by external wing buds) were collected in groups of 15 as second-day fourth instars (the last instar before adulthood) or second-day adults and immediately frozen in liquid nitrogen. This process was repeated three times to obtain three biological replicates. Unwinged female aphids were collected in the same manner, with the exception that their mothers were not crowded. All aphids were harvested during the same time of the day ( 11:00 AM) to avoid the effects of photoperiod on the collections.

To collect winged and unwinged males, we induced asexual females to produce sexual females and males under photoperiod conditions of 13 hours of light alternating with 11 hours of dark at $16^{\circ} \mathrm{C}$. Offspring were transferred to individ- 
ual dishes, monitored for molting, and collected in groups of 15 as second-day fourth instars (identified by the presence or absence of external wing buds) or second-day adults. Adult males were distinguished from females by general body shape as well as external genitalia. Fourth instar males were collected by pulling out the gonads with forceps, checking for the presence of testes, and immediately freezing the whole body (plus dissected testes) in liquid nitrogen.

\section{Pea aphid cDNA microarray}

The pea aphid cDNA microarray (Wilson et al. 2006) was made in collaboration with Wayne Hunter (USDA, ARS, Fort Pierce, FL) and Nancy Moran (University of Arizona). The array consists of quadruplicate spots of 1734 unique cDNAs from the pea aphid (an estimated 10\% of coding genes) and 117 unique genes from the bacterial endosymbiont Buchnera aphidicola. The cDNAs were derived from mRNA harvested from multistage whole-body collections of parthenogenetic aphids and single-pass sequenced from the 50 end (Sabater-Munoz et al. 2006). The resulting express sequence tags (ESTs) were compiled into a unigene set, from which contigs and singletons were selected for inclusion on the array. Of the 1,734 aphid cDNAs, 105 (6.1\%) fail to return BLASTx hits against the "nr" protein database ( $e$-value cutoff of $\left.1 \times 10^{-3}\right)$.

\section{Material preparation and hybridization}

Total RNA was isolated using the SV Total RNA Isolation Kit (Promega, Madison, WI). Twenty micrograms of total RNA was used to produce Alexa Fluor-labeled cDNA with the SuperScript Plus Direct cDNA Labeling System (Invitrogen, Carlsbad, CA).

Slides were UV-cross linked with 6,000 $\mu \mathrm{J}$ and prehybridized for 1 hour in $3 \times$ SSC, $0.1 \%$ sodium dodecyl sulfate (SDS) and $1 \%$ blocking agent (Roche, Nutley, NJ). Slides were boiled for 2 minutes before drying. Labeled cDNA was hybridized to slides under lifter slips (Erie Glass, Portsmouth, $\mathrm{NH}$ ) overnight at $65^{\circ} \mathrm{C}$ in a hybridization buffer containing $3 \times$ SSC, $0.1 \%$ SDS, $20 \mathrm{mM}$ Hepes, $13.6 \mu \mathrm{g}$ tRNA and $40 \mu \mathrm{g}$ polyA RNA. All dye handling and hybridizations were conducted in an ozone free room. In each case, the experimental sample was labeled with Alexa Fluor 647 and hybridized against a common referent, which was prepared from a large-scale RNA isolation of parthenogenetic aphids at all stages of development and labeled with Alexa Fluor 555.

Slides were scanned using an Agilent DNA Microarray Scanner (Agilent Technologies, Santa Clara, CA) and preprocessed images of each of the two fluorescent channels were created as separate image files. GenePix v 5.0 (Molecular Devices, Sunnyvale, CA) was used to grid slides, flag abnormal features, and to produce a results file of spot features. The data discussed in this publication have been deposited in NCBIs Gene Expression Omnibus (GEO, http:// www. ncbi.nlm.nih.gov/geo) and are accessible through GEO Series accession number GSE8008.

\section{Analysis of microarray data}

Before analysis, data were dye-normalized by multiplying data in one channel with a constant such that the mean of $\log 2$ ratios on the array would be zero. The normalization constant was calculated using only well-measured spots whose percentage of pixels with intensities greater than one standard deviation above the background intensity was > $65 \%$ for both channels. All data were then filtered to exclude spots that were flagged automatically by GenePix or manually during feature extraction, had pixel-to-pixel regression correlations $<0.6$, or a mean signal intensity in either channel of $<160$.

\section{Principal component analysis (PCA) of aphid types}

PCA of the variation between aphid types was performed in Acuity 4.0 (Molecular Devices) using normalized and filtered data by taking the arithmetic mean of log2 ratios for each transcript (against the common referent) for each aphid type.

\section{Identification of genes with differential transcript accumu- lation}

Transcripts whose accumulation differed significantly between aphid types were identified using a modified $t$-test (SAM, Tusher et al. 2001). Using normalized and filtered data, $\log 2$ ratios for each gene (against the common referent) for each aphid type (three replicates each) were tested using a $q$-value threshold of 0.10 (Storey 2002). To confirm the identity of genes whose transcripts appeared to accumulate differentially between winged and unwinged morphs, we submitted the polymerase chain reaction (PCR) products that were used to spot the array to be sequenced. If sequencing was unsuccessful on these products, we sequenced the original cDNA clone. Unconfirmed genes (six total) were culled from future analyses.

\section{Gene clustering}

Identified transcripts for each developmental stage and sex were separately clustered in a hierarchical manner (Eisen et al. 1998) by non-centered Pearson's correlation using data from all three replicates for both winged and unwinged morphs.

\section{Overrepresentation analysis}

To identify functionally related groups of genes, we used the program Blast2Go (Conesa et al. 2005) to identify homologs of the aphid ESTs and to link them to their gene ontology (GO) terms. We then used Fisher's exact test as part of the GOSSIP Package (Bluthgen et al. 2005), itself built into Blast2Go, to look for overrepresentation of GO terms among identified lists of genes relative to the whole microarray.

\section{Real-time quantitative PCR (qPCR)}

We used real-time qPCR to quantify the relative transcript accumulation between winged and unwinged morphs for transcripts identified by the microarray analyses. We tested transcripts that showed differential accumulation only in fourth instars (5), only as adults (11), and at both life stages (12). Primers to target transcripts were designed with Primer Express v. 2 (Applied Biosystems, Foster City, CA) using the associated EST (supplementary Table S4). Primer specificity was verified before qPCR using disassociation curve analysis. qPCR was conducted on three biological replicates for each aphid type. Because sufficient RNA was not available after the microarray experiments, we collected new tissue and extracted RNA twice each for winged fourth females, unwinged 
fourth females, unwinged fourth males, and unwinged fifth males, and once each for winged fourth males and winged fifth males. One microgram of RNA from each sample was reverse transcribed using the high capacity cDNA reverse trancription kit (Applied Biosystems) and $2.5 \mu$ added to 25 $\mu \mathrm{l}$ PCR reactions using Power SYBR Green Master Mix (Applied Biosystems). Samples were split into technical replicates and run on an ABI Prism 7900HT (Applied Biosystems) for 10 minutes at $95^{\circ} \mathrm{C}$, followed by 40 cycles of 15 seconds at $95^{\circ} \mathrm{C}$, followed by 1 minute at $60^{\circ} \mathrm{C}$. Using the Sequence Detection System v. 2.1 (Applied Biosystems), the resulting data within each sample were normalized against an endogenous control $\left(\Delta C_{\mathrm{T}}\right)$, non-ATPase subunit 12 of the $26 \mathrm{~S}$ proteasome (CF587510), selected because of its relative stability across all microarray experiments (more stable, in fact, than the commonly used endogenous control GADPH, CN586865). The $\Delta C_{T}$ values for winged samples were then calibrated against respective unwinged samples within each of the four aphid classes as determined by sex and instar $\left(\Delta \Delta C_{T}\right)$ and this value was used to calculate fold-difference in expression between winged and unwinged morphs.

\section{RESULTS AND DISCUSSION}

Pea aphids are born as first instar nymphs and pass through three additional instars before the final adult molt. In females, winged development appears to be the default developmental pathway, as all female nymphs possess wing buds and flight musculature in the first instar (Tsuji and Kawada 1987). By the second instar, the winged and unwinged developmental pathways have diverged; at this stage, the wing buds of future unwinged females have degenerated and the development of flight musculature has ceased (A. Ishikawa and S. Hongo, personal communication). In males, the developmental divergence has not been as carefully characterized, although it presumably commences before the appearance of subtle external morphological differences in the third instar. Below we describe genome-wide differences in transcript accumulation that accompany the events occurring in both sexes after the winged and unwinged forms diverge. We extracted RNA from whole animals of eight different types: winged and unwinged fourth instar nymphs of both sexes, as well as winged and unwinged adults of both sexes. Three samples of each type, 24 total, were hybridized to a pea aphid cDNA microarray possessing probes to an estimated $10 \%$ of the coding genome (1,734 unique genes).

\section{PCA reveals common differential transcript accumulation between female and male wing morphs}

We used PCA to investigate how the eight aphid types are distributed with respect to the major axes of variation. PCA reveals that principal component 1 (PC1), which accounts for $44 \%$ of the variance in the data, separates winged and unwinged morphs within each sex and developmental stage (Figure 1). PC1 also partially separates sexes for each wing morph and developmental stage. PC2, which accounts for $14 \%$ of the variance, primarily reflects differences between fourth instars and adults.

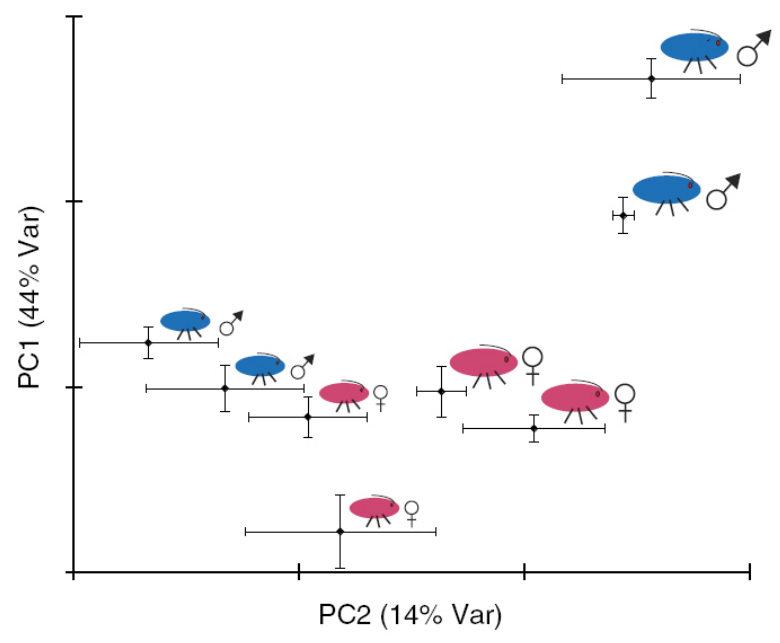

Fig. 1. Distribution of aphid types along two principal components. Aphid types are positioned along the first two principal components according to their transcriptional profiles using the entire filtered data set. The percentage of variance captured by each $\mathrm{PC}$ is indicated along its axis. The mean value along each principal component for each aphid type (indicated to the upper right of each data point) is shown, with error bars indicating one standard error. A similar distribution of aphid types was found when only genes exhibiting significant differential transcript accumulation were used for PCA (data not shown). Females are depicted in pink; males are in blue. Fourth instars (with and without wing buds) are smaller than adults (with and without wings).

The distribution of aphid types along PC2 suggests that common differences in transcript accumulation between developmental stages are found among winged and unwinged females and males. Similarly, the distribution of aphid types along PC1 suggests that common differences in transcript accumulation between sexes are found among winged and unwinged aphids as fourth instars and adults. Consistent with our expectations, the distribution of aphid types along PC1 also suggests that common differences in transcript accumulation between wing morphs are found among females and males (i.e., the polyphenism and the polymorphism) as fourth instars and adults. Contrary to our expectations, the magnitude of these differences is greater for adult males than for adult females (see relatively greater distance separating adult male wing morphs along PC1, Figure 1). Below we discuss the transcripts that exhibit significant differential transcript accumulation between the winged and unwinged morphs. Analysis of significant differential transcript accumulation between sexes and developmental stages is provided as supplementary material (supplementary Figure S1).

Genes exhibiting differential transcript accumulation between winged and unwinged morphs

We identified genes that exhibit significant differential transcript accumulation $(q<0.10)$ between winged and unwinged 
morphs of each sex and developmental stage (Figure 2A). We performed qPCR on a subset of these transcripts to verify the differential transcript accumulation observed with the micro-array (supplementary Table S3). As a general measure of consistency, we determined the correlation between the average fold difference of the microarray data and the qPCR data to be $0.76(n=17), 0.64(n=17), 0.99(n=23)$, and 0.99 $(n=23)$, for fourth instar females, fourth instar males, adult females, and adult males, respectively. We thus regard the overall patterns of the microarray data to be reliable.

In fourth instar nymphs, similar numbers of transcripts differentially accumulate between wing morphs in females and males (141 and 131, $q<0.10)$. Likewise, the overall magnitudes of these fourth instar differences in transcript accumulation are similar in both sexes.

In contrast, adult males exhibit a much larger number of differentially accumulated transcripts between wing morphs than do adult females (353 and 142 transcripts, respectively). Further, the overall magnitudes of these differences are much greater for males than for females (paired $t$-test, $P=2.2 \times 10^{-5}$; also note greater contrast along the yellow to blue spectrum of fold differences shown for adult males, Figure 2A). This is due primarily to an increase in transcript levels in winged males upon maturation rather than a decrease in transcript
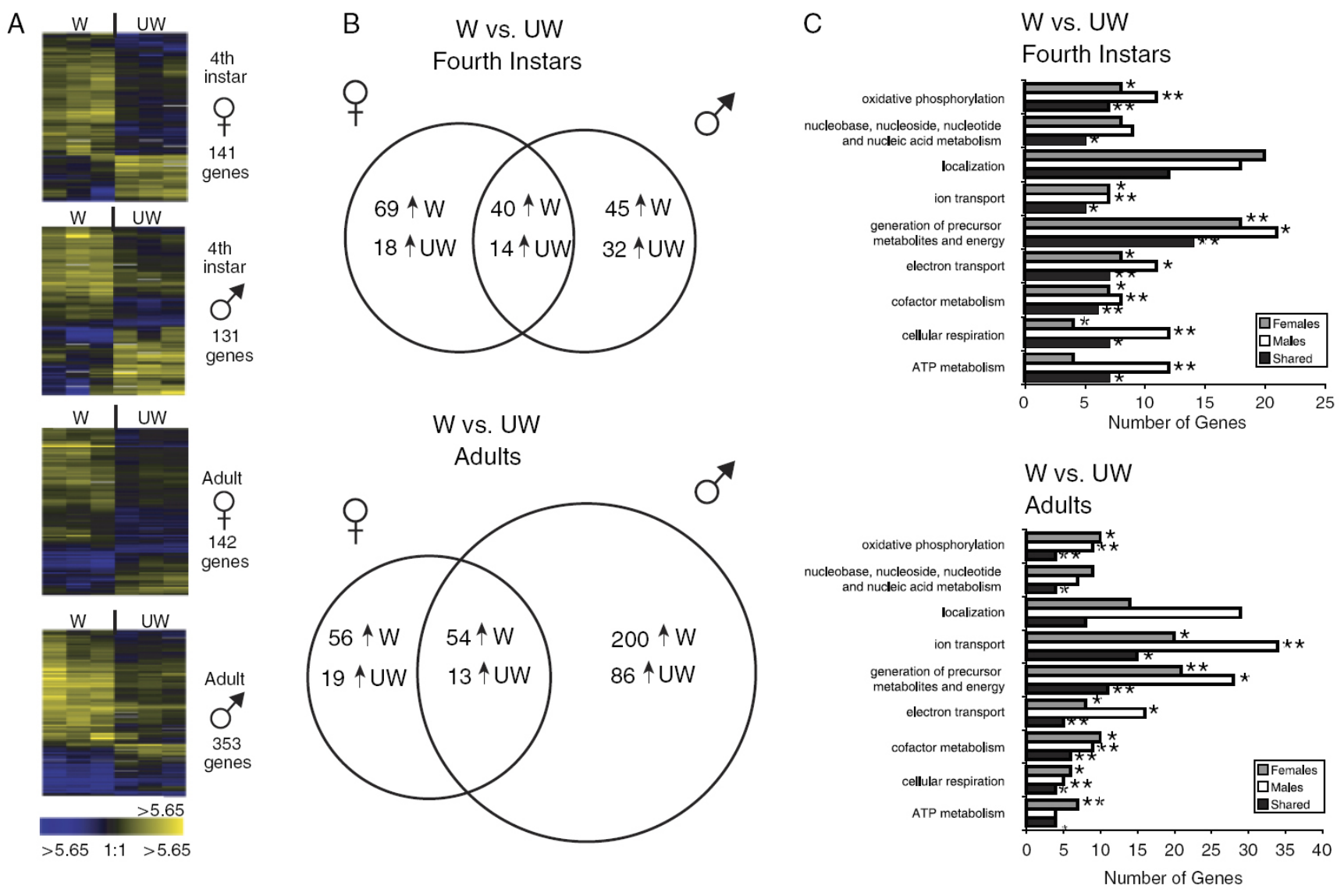

Fig. 2. Genes with differential transcript accumulation between winged and unwinged morphs of both sexes and both developmental stages. (A) Hierarchical clusters of transcripts that differentially accumulate between winged and unwinged morphs are shown. For females and males, fourth instars and adults, each column represents a separate microarray experiment (three replicates for each aphid type) and each row represents a transcript showing a significant $(q<0.10)$ difference in levels between the winged morph (left three experiments of each cluster) and unwinged morph (right three experiments of each cluster). Colors represent fold change against a common referent such that lesser accumulation is shown in blue and greater accumulation is shown in yellow (see fold change scale bar at bottom). Blanks indicate missing data. (B) Venn diagrams depicting the sets of transcripts with significant $(q<0.10)$ differential accumulation. The numbers indicate the number of transcripts in each data set that are specific to each sex or shared between the sexes (overlap between circles). The circled area is roughly proportional to the number of transcripts it comprises. Arrows indicate greater transcript levels in either the winged (W) or unwinged (UW) morph. (C) Number of transcripts with differential accumulation between winged and unwinged morphs that are associated with particular biological process gene ontology (GO) terms (only GO terms associated with at least four transcripts are shown). Asterisks indicate that a GO term was significantly overrepresented in the data set $\left({ }^{*} q<0.10,{ }^{* *} q<0.001\right)$. 
Table 1. Transcripts showing differential accumulation between winged and unwinged morphs of males and females as fourth instars and as adults $(\%<0.10)$

\begin{tabular}{|c|c|c|c|c|}
\hline $\begin{array}{l}\text { Accession } \\
\text { number }{ }^{1}\end{array}$ & BLASTx hit name ${ }^{2}$ & $\begin{array}{c}\text { Fold-difference } \\
\text { females }^{3,4}\end{array}$ & $\begin{array}{c}\text { Fold-difference } \\
\text { males }^{4}\end{array}$ & $\begin{array}{c}\text { Mitochondrially } \\
\text { localized }\end{array}$ \\
\hline CN584796 & Similar to flightin & $5.62,21.14$ & $3.93,25.77$ & $\mathrm{~N}$ \\
\hline CN584859 & Similar to ENSANGP00000024901 & $2.55,1.68$ & $1.57,1.44$ & $\mathrm{Y}$ \\
\hline CN586153 & Cytochrome c oxidase polypeptide IV & $2.27,1.72$ & $2.08,1.62$ & $\mathrm{Y}$ \\
\hline CN582783 & Similar to ENSANGP00000013101 & $1.96,1.94$ & $1.82,2.79$ & Y \\
\hline CN583311 & Putative ATP synthase $\beta$ subunit & $1.91,1.53$ & $1.92,1.53$ & $\mathrm{Y}$ \\
\hline CN583570 & Mitochondrial ATP synthase $\mathrm{O}$ subunit & $1.85,1.98$ & $2.27,1.79$ & Y \\
\hline CF588410 & No BLASTx hits & $1.84,1.56$ & $1.95,1.60$ & $\mathrm{U}$ \\
\hline CN586564 & ATP synthase c-subunit-like & $1.68,1.97$ & $2.23,1.89$ & $\mathrm{Y}$ \\
\hline CF588258 & Rieske iron-sulfur protein 1 & $1.62,1.89$ & $1.78,1.97$ & $\mathrm{Y}$ \\
\hline CN587001 & ATP synthase c-subunit-like & $1.58,1.84$ & $2.06,2.07$ & $\mathrm{Y}$ \\
\hline CN585745 & Similar to CG12814-PA & $1.49,1.44$ & $1.77,1.46$ & $\mathrm{U}$ \\
\hline CN586468 & ATP synthase A chain subunit 6 & $1.49,1.57$ & $1.86,3.52$ & $\mathrm{Y}$ \\
\hline CN586325 & Similar to $C H O R D$ & $1.46,1.28$ & $1.74,1.35$ & $\mathrm{~N}$ \\
\hline CF587936 & Trehalase & $0.47,0.60$ & $0.47,0.55$ & $\mathrm{~N}$ \\
\hline
\end{tabular}

${ }^{1}$ For the pea aphid ESTs representing the cDNAs on the microarray.

${ }^{2}$ Obtained from the highest BLASTx hit with an $e$-value cutoff of $1 \times 10^{-10}$.

${ }^{3}$ Transcripts are sorted in descending order by fold difference in fourth instar females.

${ }^{4}$ The first number is the fold difference in the fourth instars; the second is the fold difference in adults. $\mathrm{Y}$, yes; N, no; U, unknown.

levels in unwinged males (data not shown). The larger number and greater magnitude of differences in transcript levels between adult male morphs relative to adult female morphs indicates that transcript levels in males diverge substantially as they transition from fourth instars to adults, underlying the unexpected observation of greater separation of adult male morphs relative to adult female morphs along PC1 (Figure 1).

In both sexes and both stages, most of the differentially accumulated transcripts show higher levels in the winged morph rather than the unwinged morph (Figure 2B, Supplementary Tables S1 and S2). In both fourth instars and adults, much of this differential accumulation is found in both females and males (i.e., 54 transcripts for fourth instars and 67 transcripts for adults differentially accumulate in the same direction between the wing morphs in both sexes, overlap in Figure 2B). Fourteen transcripts differentially accumulate between winged and unwinged morphs regardless of sex or developmental stage (Table 1). Only one transcript in this set, trehalase, shows higher levels in the unwinged morphs; the remaining transcripts show higher levels in the winged morphs. The identification of a substantial number of genes that show higher transcript levels in the winged morphs of both females and males suggests that the polyphenism and polymorphism rely upon similar gene products to develop and maintain the winged state.

\section{Function of differentially accumulated transcripts: muscles}

Among the genes showing higher transcript levels in winged morphs are homologs of genes whose products are known muscle components: flightin, muscle actin, and myosin 1 light-chain protein. Pea aphid flightin (CN584796; $e$-value $=2$ $\times 10^{-17}$ to Drosophila melanogaster flightin) in particular exhibits the greatest differential transcript accumulation in winged morphs of both sexes at both developmental stages (Table 1 and Supplementary Table S3). In D. melanogaster, flightin's gene product is a myosin rod-binding component of indirect flight muscles (Vigoreaux et al. 1993; Ayer and Vigoreaux 2003). It is associated with the assembly of indirect flight muscle thick filaments during late pupal development and necessary for thick filament stability of these muscles in adult flies; in flightin mutants, the indirect flight muscles degenerate following the initiation of contraction (Reedy et al. 2000). The disproportionately high levels of flightin transcript in winged versus unwinged morphs likely result from the presence and absence of indirect flight muscles in winged and unwinged morphs, respectively. Because of flightin's role in maintaining flight muscles, however, it is possible that its differential transcript accumulation also plays a causal role in the morphological divergence of the wing morphs.

Function of differentially accumulated transcripts: energy production

Of the genes showing higher transcript levels in the winged morphs of both sexes (i.e., genes found in the overlap of Figure $2 \mathrm{~B})$, many are predicted to function in the mitochondria, including both nuclear-and mitochondrial-encoded genes. The proportion of transcripts with mitochondrial function is larger in fourth instars than adults (27 out of 40 for fourth in- 
stars, 21 out of 54 for adults; supplementary Tables S1 and S2). In contrast, of the genes that show higher transcript levels in the unwinged morphs of both sexes, none are known to have mitochondrial function (supplementary Tables S1 and S2). Consistent with differential mitochondrial function, biological process GO terms associated with energy production are significantly overrepresented among GO terms associated with the genes that show differential transcript accumulation between wing morphs of both sexes (asterisks, Figure 2C). We observed similar results when we extended the analysis to the male and female data sets, which included the transcripts exhibiting shared differential accumulation (Figure $2 \mathrm{C}$ ).

The observation that genes involved in energy production show increased transcript levels in winged morphs relative to unwinged morphs is consistent with the metabolic requirements for the production of wings and muscles as fourth instars, and the capability of flight and the maintenance of flight muscles as adults (Zera and Denno 1997).

\section{Concluding remarks}

We have characterized genome-wide changes in transcript accumulation that are associated with the developmental divergence of the winged and unwinged morphs of female and male pea aphids. These data provide a foundation for future studies on the molecular and physiological basis of tradeoffs in life-history evolution. We have used these data to test the hypothesis that there are similarities in differential transcript accumulation, and possibly gene regulation, between the female wing polyphenism and the male wing polymorphism. We find that many transcripts differentially accumulate in both sexes, as fourth instar nymphs and as adults (Figures 1 and $2 \mathrm{~B}$ ). This indicates that similar gene products are associated with the divergent development and maintenance of wing morphs in the polyphenism and the polymorphism. Further, differential transcript accumulation between wing morphs that appears specific to the polymorphism or polyphenism nevertheless overlaps in function. Surprisingly, the extent of differential transcript accumulation between wing morphs appears to be more pronounced in adult males (Figures 1 and 2), contrary to our expectations based on differences in the morphology and considerations of reproductive investment. Although it is possible that this observed disparity between males and females was biased by a swamping effect of RNA from the daughter embryos of harvested females, the data support the hypothesis that male wing morphs, like female wing morphs, are subject to selective trade-offs between reproduction and dispersal.

Whereas the evolutionary relationship between wing polyphenism and polymorphism is not well understood, the winged state is ancestral for both females and males (reviewed in Braendle et al. 2006). The differences in morphology and behavior between wing morphs that are shared between females and males may be related to the shared patterns of transcript accumulation that we observe. Males may have coopted the genetic and developmental programs used by females to reallocate resources from dispersal to reproduction.

\section{Acknowledgments}

We would like to thank Phat Dang and Wayne Hunter for generating the original EST sequence data and for providing cDNA clones; Alex Wilson, Nancy Moran, and Ryan Sprissler for assistance with the design and printing of the microarray; Donna Storton, John Matese, and Kara Dolinski for assistance with hybridizations and data analysis; and Ryan Bickel, Amy Caudy, Tony Frankino, Manuel Llinás, Erin Smith, Dayalan Srinivasan, and Saeed Tavazoie and two anonymous reviewers for comments on the manuscript. J. A. B. and G. K. D. were supported by NRSA postdoctoral fellowships from the NIH. D. L.S. was supported bythe NIH (Grant GM6362201), Princeton University and the David and Lucile Packard Foundation.

\section{REFERENCES}

Ayer, G., and Vigoreaux, J. O. 2003. Flightin is amyosinrod binding protein. Cell Biochem. Biophys. 38: 41-54.

Blackman, R. L. 1987. Reproduction, cytogenetics and development. In: A. K. Minks and P. Harrewijn (eds.). Aphids: Their Biology, Natural Enemies \& Control. Elsevier, Amsterdam, pp. 163-195.

Bluthgen, N., Brand, K., Cajavec, B., Swat, M., Herzel, H., and Beule, D. 2005. Biological profiling of gene groups utilizing Gene Ontology. Genome Informatics 16: 106-115.

Braendle, C., Caillaud, M. C., and Stern, D. L. 2005a. Genetic mapping of aphicarus - a sex-linked locus controlling a wing polymorphism in the pea aphid (Acyrthosiphon pisum). Heredity 94: 435-442. Braendle, C., Davis, G. K., Brisson, J. A., and Stern, D. L. 2006. Wing dimorphism in aphids. Heredity 97: 192-199.

Braendle, C., Friebe, I., Caillaud, M. C., and Stern, D. L. 2005b. Genetic variation for an aphid wing polyphenism is genetically linked to naturally occurring wing polymorphism. Proc. Roy. Soc. Lond. B 272: 657665.

Caillaud, M. C., Boutin, M., Braendle, C., and Simon, J.-C. 2002. A sexlinked locus controls wing polymorphism in males of the pea aphid, Acyrthosiphon pisum (Harris). Heredity 89: 346-352.

Collins, J. P., and Holomuzki, J. R. 1984. Intraspecific variation in diet within and between trophic morphs in larval tiger salamanders (Ambystoma-Tigrinum-Nebulosum). Can. J. Zool. 62: 168-174.

Conesa, A., Gotz, S., Garcia-Gomez, J. M., Terol, J., Talon, M., and Robles, M. 2005. Blast2Go: a universal tool for annotation, visualization and analysis in functional genomics research. Bioinformatics 21: 3,6743,676 .

Dixon, A. F. G., and Howard, M. T. 1986. Dispersal in aphids, a problem in resource allocation. In W. Danthanarayana (ed.). Insect Flight: Dispersal and Migration. Springer-Verlag, Berlin, pp. 145-151.

Eisen, M. B., Spellman, P. T., Browndagger, P. O., and Botstein, D. 1998. Cluster analysis and display of genome-wide expression patterns. Proc. Nat. Acad. Sci. USA 95: 14,863-14,868.

Ghanim, M., Dombrovsky, A., Raccah, B., and Sherman, A. 2006. A microarray approach identifies ANT, OS-D and takeout-like genes as differentially regulated in alate and apterous morphs of the green peach aphid Myzus persicae (Sulzer). Insect Biochem. Mol. 36: 857-868.

Hales, D. F., Wilson, A. C. C., Sloane, M. A., Simon, J.-C., Legallic, J.-F., and Sunnucks, P. 2002. Lack of detectable genetic recombination on the $\mathrm{X}$ chromosme during the parthenogenetic production of female and male aphids. Genet. Res. Camb. 79: 203-209. 
Hazell, S. P., Gwynn, D. M., Ceccarelli, S., and Fellowes, M. D. E. 2005. Competition and dispersal in the pea aphid: clonal variation and correlations across traits. Ecol. Entomol. 30: 293-298.

Kawada, K. 1987. Polymorphism and morph determination. In: A. K. Minks and P. Harrewijn (eds.). Aphids, Their Biology, Natural Enemies and Control. Elsevier, Amsterdam, pp. 255-268.

Kring, J. B. 1977. Structure of the eyes of the pea aphid, Acyrthosiphon pisum. Annals Ent. Soc. Am. 70: 855-860.

Markkula, M. 1963. Studies of the pea aphid, Acyrthosiphon pisum Harris (Hom., Aphididae), with special reference to the differences in the biology of the green and red forms. Ann. Agriculturae Fenniae 2: 1-30.

Miyazaki, M. 1987. Forms and morphs of aphids. In: A. K. Minks and P. Harrewijn (eds.). Aphids, Their Biology, Natural Enemies and Control. Elsevier, Amsterdam, pp. 163-195.

Muller, C. B., Williams, I. S., and Hardie, J. 2001. The role of nutrition, crowding, and interspecific interactions in the development of winged aphids. Ecol. Ent. 26: 330-340.

Nijhout, H. F. 2003. Development and evolution of adaptive polyphenisms. Evol. Dev. 5: 9-18.

Reedy, M. C.,Bullard,B., and Vigoreaux, J. O. 2000. Flightin is essential for thick filament assembly and sarcomere stability in Drosophila flight muscles. J. Cell Biol. 151: 1,483-1,499.

Sabater-Munoz, B., et al. 2006. Large-scale gene discovery in the pea aphid Acyrthosiphon pisum (Hemiptera). Genome Biol. 7: R21.1-R21.11.

Sack, C., and Stern, D. L. 2007. Sex and death in the male pea aphid: Acyrthosiphon pisum: The life-history effects of a wing dimorphism. J. Insect Sci. in press.

Smith, M. A. H., and MacKay, P. A. 1989. Genetic variation in male alary dimorphism in populations of the pea aphid, Acyrthosiphon pisum. Entomol. Exp. Appl. 51: 125-132.

Smith, T. B. 1993. Disruptive selection and the genetic basis of bill size polymorphism in the African finch Pyrenestes. Nature 363: 618-620.

Storey, J. 2002. A direct approach to false discovery rates. J. Roy. Stat. Soc., Ser. B 64: 479-498.

Sutherland, O. R. W. 1969. The role of crowding in the production of winged forms by two strains of the pea aphid, Acyrthosiphon pisum. J. Insect Phys. 15: 1,385-1,410.

Takaoka, I. 1973. Review on emergence of alate virginoparous females of Aphididae. Bull. Okayama Tobacco Exp. Station 32: 101-135 (in Japanese).

Tsuji, H., and Kawada, K. 1987. Development and degeneration of wing buds and indirect flight muscles in the pea aphid (Acyrtosiphon pisum (Harris)). Jpn. J. Appl. Ent. Zool. 31: 247-252.

Tusher,V. G., Tibshirani, R., and Chu, G. 2001. Significance analysis of microarrays applied to the ionizing radiation response. Proc. Nat. Acad. Sci. USA 24: 5,116-5,121.

Via, S. 1992. Inducing the sexual forms and hatching the eggs of pea aphids. Ent. Exp. Appl. 65: 119-127.

Vigoreaux, J. O., Saide, J. D., Valgeirsdottir, K., and Pardu, M. L. 1993. Flightin, a novel myofibrillar protein of Drosophila stretch-activated muscles. J. Cell Biol. 121: 587-598.

West-Eberhard, M. J. 2003. Developmental Plasticity and Evolution. Oxford University Press, New York.

Wilson,A. C. C., Dunbar, H. E., Davis, G. K., Hunter, W. B., Stern, D. L., and Moran, N. A. 2006. A dual-genome microarray for the pea aphid, Acyrthosiphon pisum, and its obligate bacterial symbiont, Buchnera aphidicola. BMC Genomics 7: 50.

Zera, A. J., and Denno, R. F. 1997. Physiology and ecology of dispersal polymorphism in insects. Ann. Rev. Ent. 42: 207-230.

\section{SUPPLEMENTARY MATERIAL}

The following supplementary material is available for this article:

Table S1. Transcripts showing differential accumulation between winged and unwinged morphs of males and females during the fourth instar $(\%<0.10)$.

Table S2. Transcripts showing differential accumulation between winged and unwinged morphs of males and females as adults $(\%<0.10)$.

Table S3. Quantitative PCR results.

Table S4. ESTs and their associated forward and reverse qPCR primers.

Figure S1. Numbers of genes showing significant differences in transcript levels between developmental stages and sexes. The number of transcripts showing significantly $(q<$ 0.10) higher levels (small arrow up) and lower levels (small arrow down) of transcript in adults relative to fourth instars (horizontal comparisons) and males relative to females (vertical comparisons) are indicated for winged (A) and unwinged (B) morphs. Most of the genes that show differential levels of transcript between stages decrease transcript levels during the transition from fourth instar to adult. This trend is true for all four aphid types, although the number of genes showing significant differences in transcript levels between stages are greater for males than for females (893 and 340 versus 83 and 143 genes) and greater for winged than for unwinged males (893 versus 340 genes). The number of genes showing significantly different transcript levels between females and males increases substantially upon maturation to adulthood. This increase is greater for winged aphids ( 0 genes as fourth instars to 1,155 genes as adults, representing two-thirds of the genes on our array) than for unwinged aphids (228 genes as fourth instars to 881 genes as adults). Although sexual differences are fewer in the fourth instars of both wing morphs, the lack of any detectable differences between female and male winged fourth instars most likely reflects the observed delay in the developmental rate of winged versus unwinged morphs (Dixon and Howard 1986). In contrast to the relatively large number of genes showing a decrease in transcript levels during the fourth instar-adult transition, sexual differences in transcript levels were approximately equally divided between those that are higher in females and those that are higher in males.

Colored numbers indicate significant overrepresentation of biological process gene ontology terms relative to all genes on the array. Red indicates over-representation for genes involved in protein synthesis whose products are components of ribosomes $(q<0.05)$. Of these, genes showing lower transcript levels in unwinged male adults relative to fourth instars are also enriched for cuticle components $(q<0.05)$, while the group of genes lower transcript levels in winged and unwinged adult males relative to adult females is additionally enriched for transcripts whose products are found in intracellular vesicle coats $(q<0.10)$. Green indicates enrichment for transcripts involved in transition metal ion binding, oxidoreductase activity, and carbohydrate metabolism $(q<$ 0.05 ) among genes showing higher transcript levels in unwinged adult males relative to females.

The fact that genes with higher levels of transcript in male fourth instars relative to adults, and females relative to 
males, are enriched for genes involved in protein synthesis may reflect, respectively, a need to accommodate the divergent gene expression that males experience upon maturation to adulthood and the demands of asexual reproduction and embryogenesis placed on fourth instar and adult females.

This material is available as part of the online article from: http://www.blackwell-synergy.com/doi/abs/10.1111/j.
1525-142X.2007.00170.x (This link will take you to the article abstract).

Please note: Blackwell Publishing is not responsible for the content or functionality of any supplementary materials supplied by the authors. Any queries (other than missing material) should be directed to the corresponding author for the article. 